\title{
Artificial Biosynthetic Pathway for an Unnatural Terpenoid with an Iridium- containing P450
}

\author{
Jing Huang, ${ }^{1,2 \dagger}$ Zhennan Liu, ${ }^{3,4 \dagger}$ Brandon J. Bloomer, Douglas S. Clark, ${ }^{5,6^{*}}$ Aindrila
} Mukhopadhyay, ${ }^{1,2,7^{*}}$ Jay D Keasling,,$^{1,2,8,9,10,11,12^{*}}$ John F. Hartwig ${ }^{3,4^{*}}$

${ }^{1}$ Biological Systems and Engineering Division, Lawrence Berkeley National Laboratory, Berkeley, CA 94720, USA.

${ }^{2}$ Joint BioEnergy Institute, Lawrence Berkeley National Laboratory, Emeryville, CA 94608, USA.

${ }^{3}$ Department of Chemistry, University of California, Berkeley, CA 94720, USA.

${ }^{4}$ Chemical Sciences Division, Lawrence Berkeley National Laboratory, Berkeley, CA 94720 , USA.

${ }^{5}$ Department of Chemical and Biomolecular Engineering, University of California, Berkeley, CA 94720, USA.

${ }^{6}$ Molecular Biophysics and Integrated Bioimaging Division, Lawrence Berkeley National Laboratory, Berkeley, CA 94720, USA.

${ }^{7}$ Environmental Genomics and Systems Biology Division, Lawrence Berkeley National Laboratory, Berkeley, CA 94720, USA.

${ }^{8}$ Department of Plant and Microbial Biology, University of California, Berkeley, CA 94720, USA.

${ }^{9}$ Department of Bio-engineering, University of California, Berkeley, CA 94720, USA.

${ }^{10}$ Department of Chemical and Biomolecular Engineering, University of California, Berkeley, CA 94720, USA.

${ }^{11}$ The Novo Nordisk Foundation Center for Biosustainability, Technical University of Denmark, Kongens Lyngby, Denmark.

${ }^{12}$ Synthetic Biochemistry Center, Institute for Synthetic Biology, Shenzhen Institutes for Advanced Technologies, Shenzhen, China.

$\dagger$ These authors contributed equally to this work.

*Correspondence to dsc@berkeley.edu, amukhopadhyay@1bl.gov, jdkeasling@1bl.gov, jhartwig@berkeley.edu

\begin{abstract}
Synthetic biology enables microbial hosts to produce complex molecules that are otherwise produced by organisms that are rare or difficult to cultivate, but the structures of these molecules are limited to chemical reactions catalyzed by natural enzymes. The integration of artificial metalloenzymes (ArMs) that catalyze abiotic reactions into metabolic networks could broaden the cache of molecules produced biosynthetically by microorgansms. We report the assembly of an ArM containing an iridium-porphyrin complex in the cytoplasm of a terpene producing Escherichia coli by a heterologous heme transport machinery, and insertion of this ArM into a natural biosynthetic pathway to produce an unnatural terpenoid. This work shows that synthetic biology and synthetic chemistry, incorporated together in whole cells, can produce molecules previously inaccessible to nature.
\end{abstract}


Molecules produced by natural biosynthetic pathways are the inspiration or the actual molecules in many chemical products, ranging from medicines, agrochemicals to commodity chemical and biofuels. Synthetic biology has provided access to complex molecules that would otherwise be produced by rare organisms or organisms difficult to cultivate in a laboratory, by incorporating genes and pathways involved in the biosynthesis into a genetically tractable microorganism. Although many molecules with structures that are the envy of chemists are produced by these natural or engineered organisms, the structures are still limited by the available chemical reactions catalyzed by natural enzymes. If artificial enzymes could be incorporated into such biosynthetic pathways, then microorganisms could produce unnatural products from a combination of nature's reactions and chemists' imaginations.

Recently, artificial metalloenzymes (ArMs) have been created that catalyze a series of abiotic reactions. ${ }^{1}$ These reactions include group transfers to olefins and $\mathrm{C}-\mathrm{H}$ bonds, ${ }^{2}$ cross coupling, ${ }^{3}$ asymmetric hydrogenation, ${ }^{4}$ and ring-closing olefin metathesis. ${ }^{5}$ However, most of these reactions do not occur on the types of molecules that could be produced by biosynthesis. In contrast, an iridium-containing analog of a heme enzyme catalyzes the cyclopropanation of unconjugated, hindered, terminal alkenes that are present in natural products such as terpenes. ${ }^{2 b}$ This reactivity provides the potential to create an artificial biosynthetic pathway that combines natural pathways manipulated by synthetic biology with the catalysis created by ArMs.

The assembly of artificial metalloenzymes has advanced from in vitro reconstitution of purified proteins with organometallic complexes to assembly of ArMs in the periplasm ${ }^{5 c, 6}$ or on the outermembrane ${ }^{7}$ of living cells. However, methods to assemble ArMs in more complex intracellular environments are lacking ${ }^{8}$. We hypothesized that artificial cytochrome $\mathrm{P} 450$ s containing synthetic heme derivatives could be generated in this environment by exploiting natural heme transport machinery ${ }^{9}$ and the high binding affinity of hemoproteins to heme derivatives. Thus, artificial cytochrome P450s are potential ArMs for conducting unnatural whole-cell biotransformations and for assembling artificial biosynthetic pathways.

Terpenes are the largest and most structurally diverse family of natural products ${ }^{10}$, yet are produced by relatively short biosynthetic pathways. Their core skeletons are assembled in one step and modified by a suite of tailoring enzymes including cytochrome P450s. ${ }^{11}$ While native P450s mostly catalyze oxidations of terpenes at specific positions, artificial P450s substituted with abiotic metalloporphyrins, such as Ir(Me)MPIX (MPIX is mesoporphyrin IX), can catalyze unnatural transformations $^{2,12}$ that introduce an artificial dimension to the diversification of terpene structures. For example, we have previously reported that Ir-CYP119 (CYP119 containing the $\operatorname{Ir}(\mathrm{Me}) \mathrm{MPIX}$ cofactor) catalyzes in vitro the cyclopropanation of unconjugated, hindered and terminal alkenes present in terpene natural products, such as $\beta$-pinene, carvone, and limonene. Here we report the creation of an artificial biosynthetic pathway that combines the reactivity of IrCYP119 and natural enzymes of limonene biosynthesis, thus enabling the cellular production of terpenoids that were previously inaccessible in a microbial host.

Our overall strategy for creating an artificial biosynthetic pathway by integrating a heterologous pathway with a reaction catalyzed by an artificial metalloenzyme is depicted in Fig. 1. The artificial metalloenzyme is assembled in E. coli with the aid of a cofactor transport system and overexpression of the host protein. In concert, $E$. coli is engineered with the pathway for production of a natural product that serves as the substrate for the artificial metalloenzyme. If successful, this combination of natural and unnatural reactions in the same cell would produce an unnatural derivative of a classic natural product. 


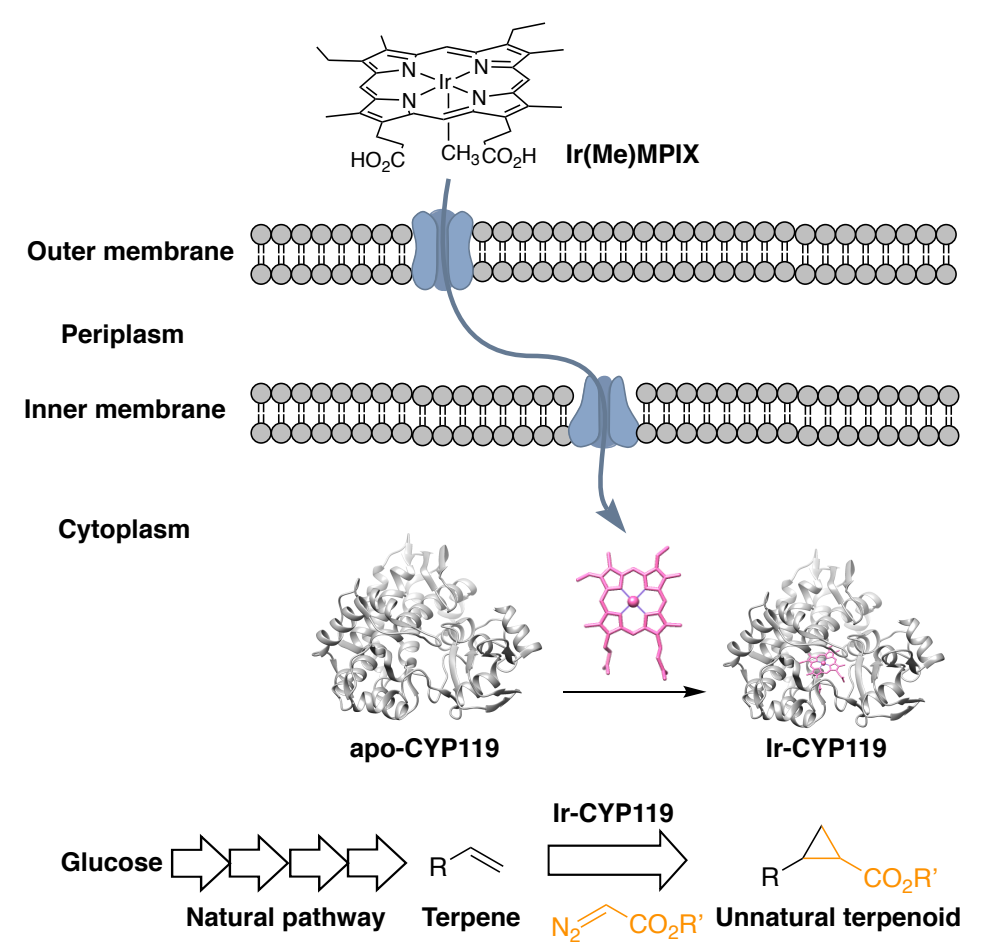

Fig. 1. A schematic presentation of an unnatural pathway containing an artificial metalloenzyme in E. coli. The artificial cofactor is imported into the cytoplasm by transporters and assembled with apo-P450. Then the holoenzyme functionalizes the terpene produced from a natural biosynthetic pathway.

The first major challenge facing this integration of an artificial metalloenzyme into a cellular biosynthetic pathway is to assemble such enzymes in the cytoplasm. Previously, we reported the in vitro construction of Ir-CYP119 by incorporation of $\operatorname{Ir}(\mathrm{Me})$ MPIX into the heme binding site of the purified, apo form of the thermophilic cytochrome P450 enzyme CYP119. To construct this artificial enzyme in a cell, transfer of the cofactor from the growth medium into the cell must occur while CYP119 is overexpressed under conditions at low temperature with low concentrations of iron to suppress the formation of heme-containing protein. Metal-substituted heme derivatives have been reported to transport across a cell membrane when the outer-membrane receptor ChuA is overexpressed. ${ }^{13}$ Thus, we first considered whether the co-expression of a heme transport system and CYP119 would enable the artificial cofactor $\operatorname{Ir}(\mathrm{Me}) \mathrm{MPIX}$ to be transported into $E$. coli. and incorporated into the apo-CYP119 C317G mutant in the cytoplasm.

To test the feasibility of assembling Ir-CYP119 and catalyzing a reaction in $E$. coli, we studied the cyclopropanation of a terpene and used the diastereoselectivity of the reaction as a parameter to distinguish any background reaction or reaction catalyzed by free cofactor from the reaction catalyzed by the assembled artificial metalloenzyme. Our previous studies on Ir-CYP119 generated from in vitro reconstitution of $\operatorname{Ir}(\mathrm{Me})$ MPIX and purified CYP119 mutants showed that the triple mutant (T213G, V254L, L155W, in addition to mutation of the axial cysteine to glycine) catalyzes the stereoselective cyclopropanation of (-)-carvone to give an $8: 1: 1: 1$ ratio of diastereomers. The major diastereomer of this reaction is a minor diastereomer of the $1: 1: 3: 3$ ratio of diastereomers formed from reaction catalyzed by the free iridium-porphyrin complex. 
Thus, a reaction that forms a mixture of diastereomers resembling the ratio produced by the pure ArM in vitro would also denote reaction by the assembled protein in the cell.

To this end, E. coli BL21(DE3) cells co-expressing the heme transporter ChuA and the CYP119 triple mutant were supplemented with $10 \mu \mathrm{M} \operatorname{Ir}(\mathrm{Me}) \mathrm{MPIX}$, and the cell pellets containing in vivoassembled Ir-CYP119 were directly employed for catalysis after re-suspension in reaction buffer. However, the diastereoselectivity (dr) of the whole-cell reaction was $1.7: 1.0: 2.7: 3.3$ (Fig. S1). The stereoselectivity indicates that the majority of the product of the whole-cell system formed from a reaction catalyzed by the free iridium-porphyrin complex, rather than that catalyzed by a properly assembled ArM. In addition, no product was detected when E. coli cultures were incubated in the absence of $\operatorname{Ir}(\mathrm{Me})$ MPIX (Fig. S1), showing that Fe-CYP119 formed by binding of natively synthesized heme to the apo-CYP119 does not catalyze the cyclopanation of (-)carvone. Consistent with this conclusion, the purified mutants of CYP119 reconstituted with hemin gave less than $2 \%$ of the cyclopropanation reaction.

To determine how to reduce interference from the background reactivity of the free iridium porphyrin, we evaluated reactions of (-)-carvone with EDA added exogeneously to cells grown in the presence of $\operatorname{Ir}(\mathrm{Me}) \mathrm{MPIX}$ in concentrations ranging from $10 \mu \mathrm{M}$ to $0.02 \mu \mathrm{M}$. These experiments showed that the $\mathrm{dr}$ of the cyclopropane product was much higher $23: 3.5: 1.0: 1.2$ when the concentration of $\operatorname{Ir}(\mathrm{Me}) \mathrm{MPIX}$ was $0.02 \mu \mathrm{M}$ (Fig. 2a). We surmise that the lower concentrations of $\operatorname{Ir}(\mathrm{Me}) \mathrm{MPIX}$ reduce the amount of product formed by a background reaction catalyzed by the cofactor unbound to CYP119. Control experiments showed that dr was low for product formed in E. coli expressing ChuA without co-expression of CYP119 or in E. coli with a control plasmid (Fig. S2); under these conditions of the control experiments, the cyclopropanation would be catalyzed by the free iridium cofactor (Fig. S2).

Having demonstrated that Ir-CYP119 assembles in vivo and that this enzyme catalyzes cyclopropanation of a terpene with high diastereoselectivity, we sought to combine this assembly and catalysis with a native biosynthetic pathway to produce an unnatural terpene. However, initial attempts to express a heterologous biosynthetic pathway to form limonene in concert with expression of CYP119 regulated by a T7 promoter caused the titer of limonene to decrease drastically from $188 \mathrm{mg} / \mathrm{L}$ without co-expression of CYP119 to $28 \mathrm{mg} / \mathrm{L}$ with co-expression (Fig. S3). Although high-level production of CYP119 is desired for maximal reactivity, it appeared to pose a metabolic burden that hinders the production of limonene.

These results show that a method is needed to incorporate $\operatorname{Ir}(\mathrm{Me}) \mathrm{PIX}$ efficiently into CYP119, even under conditions with low expression levels of this protein. Demonstrating this need, the reaction of exogeneously added carvone with EDA conducted after co-expression of ChuA and CYP119 with a weaker lacUV5 promoter formed the cyclopropane with low stereoselectivity because the dominant reaction occurred with unbound $\operatorname{Ir}(\mathrm{Me}) \mathrm{MPIX}$ (Fig. S4). However, replacement of the ChuA transporter with the alternative heme transport system encoded by the hug operon ${ }^{14}$ led to an increase in the efficiency of assembly of the Ir-CYP119. Reactions of (-)carvone with EDA conducted in E. coli expressing the HUG transport system, in concert with CYP119 (lacUV5), formed the cyclopropane product with a high $\mathrm{dr}$ of $82 \%(24: 3.1: 1.0: 1.2)$ under conditions that are identical to those of the reactions with cells possessing the ChuA transporter (Fig. 2b). 


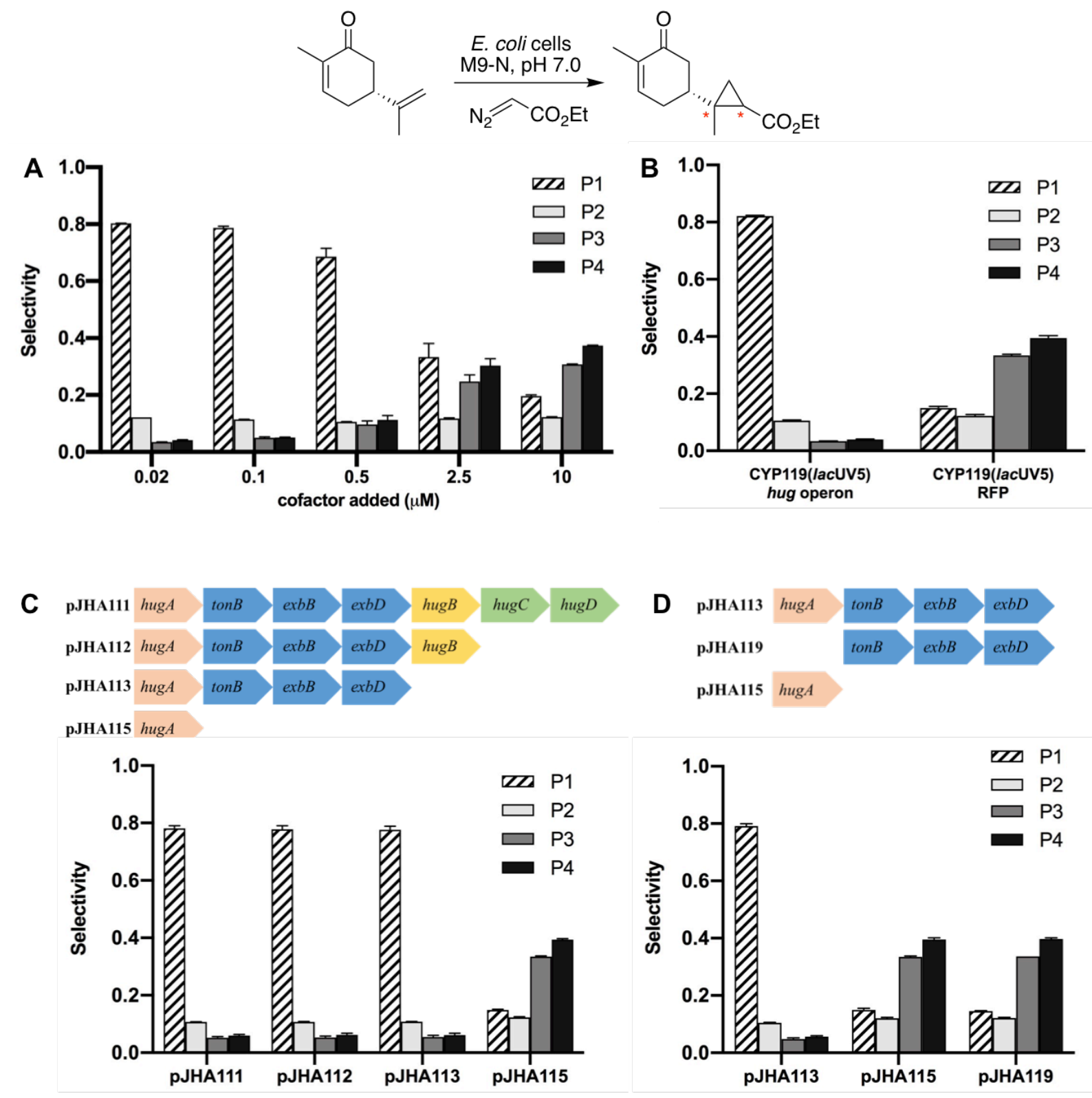

Fig. 2. Whole cell reactivity of Ir-CYP119 for the cyclopropanation of (-)-carvone. P1-P4 are the four diastereomeric products numbered in the order of elution by GC. Selectivity in the plots corresponds to the ratio of each diastereomer versus the total of the four diastereomeric products. (A) E. coli cells co-expressing the CYP119 mutant and ChuA were incubated with different concentrations of $\operatorname{Ir}(\mathrm{Me})$ MPIX. Decreasing the concentration of $\operatorname{Ir}(\mathrm{Me}) \mathrm{MPIX}$ increased the stereoselectivity of whole cell reactions as evidenced by the distribution of diastereomeric products P1-P4. (B) E. coli cells co-expressing CYP119(lacUV5) and hug operon could maintain the high stereoselectivity under low expression level of CYP119; E. coli cells containing CYP119(lacUV5) and an RFP-coding plasmid is shown as control. (C) The deletions of HugB, HugC, and HugD had no effect on the efficiency of cofactor uptake. (D) Both HugA and Ton complex are required for 
cofactor uptake in the hug operon. All data are shown as the average from three biological repeats, with error bars indicating 1 standard deviation.

To quantify the amount of $\operatorname{Ir}(\mathrm{Me})$ MPIX incorporated into CYP119 in cells expressing the HUG system, His-tagged proteins were purified and digested with nitric acid, and the iridium concentrations were determined by ICP-OES. In the presence of $0.2 \mathrm{ppm} \operatorname{Ir}(\mathrm{Me}) \mathrm{MPIX}$ during growth of the cells expressing HUG transporters, $22 \pm 1 \%$ of the cofactor was assembled into apoCYP119, whereas only $13 \pm 1 \%$ was incorporated into CYP119 when the protein was expressed in cells containing an empty vector lacking $h u g$ (Table S1). Although we cannot measure the amount of unbound $\operatorname{Ir}(\mathrm{Me})$ MPIX, the higher incorporation of $\operatorname{Ir}(\mathrm{Me})$ MPIX into CYP119 implies that the amount of unbound $\operatorname{Ir}(\mathrm{Me})$ MPIX is lower in the system containing the HUG transport system.

The intermembrane transport of $\operatorname{Ir}(\mathrm{Me}) \mathrm{MPIX}$ by the HUG system follows a pathway that consists of four parts : the outer membrane receptor HugA; the Ton complex including TonB, ExbB, and ExbD; the periplasmic shuttle protein $\mathrm{HugB}$; and the inner membrane $\mathrm{ABC}$ transporter including HugC and HugD. ${ }^{14}$ To determine which proteins in this heterologous system improve uptake of $\operatorname{Ir}(\mathrm{Me}) \mathrm{MPIX}$, we constructed a series of truncations of the hug operon by sequential removal of the $\mathrm{ABC}$ transporter, the periplasmic binding protein, and the energy transduction complex. We used the stereoselectivity of the cyclopropanation of (-)-carvone as a metric for assessing the efficiency of the truncated transport systems. Deletion of HugB, HugC, and HugD did not affect the stereoselectivity of the whole-cell reactions, suggesting that these parts are not essential for transport of $\operatorname{Ir}(\mathrm{Me}$ )MPIX (Fig. 2c). However, truncation of the Ton complex or expression of the Ton complex without HugA resulted in low selectivity for the cyclopropanation. These data indicate that HugA and the Ton complex, together, are required to support the efficient uptake of $\operatorname{Ir}(\mathrm{Me}) \mathrm{MPIX}$ (Fig. 2d).

Although (-)-carvone has been produced from glucose in E. coli, ${ }^{15}$ the presence of intermediates with structures similar to each other along the pathway and the low titer of the carvone produced biosynthetically would complicate analysis of the unnatural terpenoid products. Thus, we chose to combine the production of (-)-limonene with the reactivity of Ir-CYP119 because it is a structurally related monoterpene known to be produced in high titer in E. coli. ${ }^{16}$ Even though the two monoterpenes share the same carbon skeleton, the cyclopropanation of (-)-limonene catalyzed by the same Ir-CYP119 mutant in vitro occurs with a low diastereoselectivity of $1.0: 2.4: 2.3: 2.9$, but still different from the selectivity $1.0: 1.2: 3.5: 4.2$ of the reaction catalyzed by free Ir(Me)MPIX (Fig. 3a). In contrast, the cyclopropanation of limonene within the E. coli strain containing the hug operon under conditions involving low-expression levels of CYP119 in M9rich media produced the cyclopropane with a substantial $52 \% \mathrm{dr}(1.0: 3.3: 1.1: 1.0)$ (Fig. 3a). This higher diastereoselectivity in the whole-cell system can be attributed to greater stability of the enzyme in vivo than in vitro in the presence of limonene. The in vivo system minimizes the flux of substrates and product at the reaction site that can deactivate the protein. ${ }^{2 \mathrm{a}}$

Having demonstrated that stereoselective cyclopropanation of (-)-limonene occurs in vivo, we integrated this reactivity with the biosynthesis of limonene (Fig. 3b). We first assessed whether the expression of heme transporters and the artificial metalloenzyme would affect the production of limonene in E. coli containing a plasmid (pJBEI6410) encoding genes for production of limonene from glucose in high titer. We found that limonene production was not affected in $E$. coli strains harboring both the limonene plasmid (pJBEI16410) and a plasmid containing the the hug operon (pJHA047) or a plasmid containing hug and the gene encoding CYP119 (pJHA135) (Fig. 3c). The addition of $0.1 \mu \mathrm{M} \operatorname{Ir}(\mathrm{Me}) \mathrm{MPIX}$ to either strain did not affect the 
synthesis of limonene either (Fig. S6). This demonstration that limonene is produced in high titer set the stage for creation of an artificial pathway by integration of the biosynthesis of this terpene with its catalysis via the artificial metalloenzyme.

A
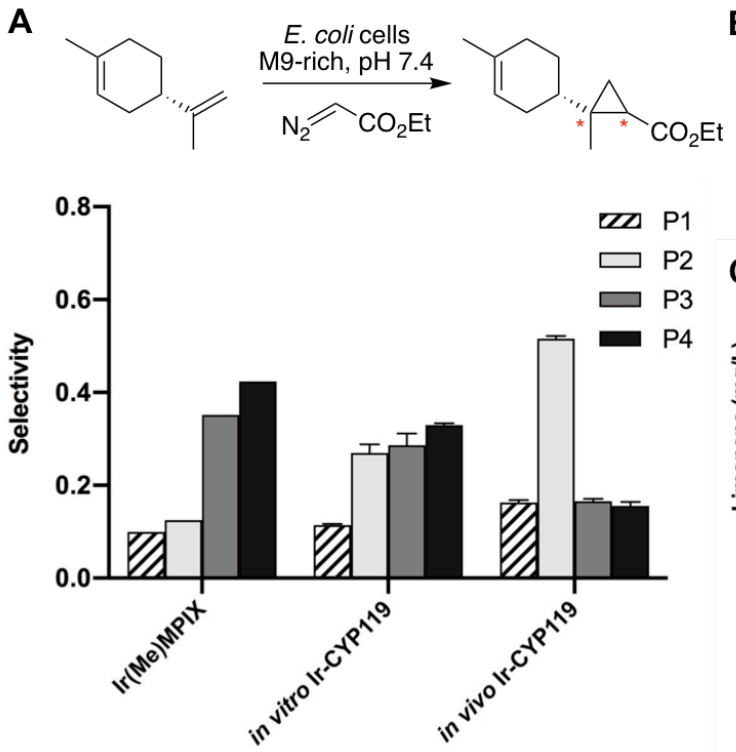

B

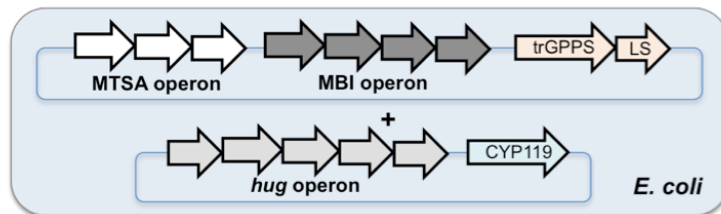

E. coli

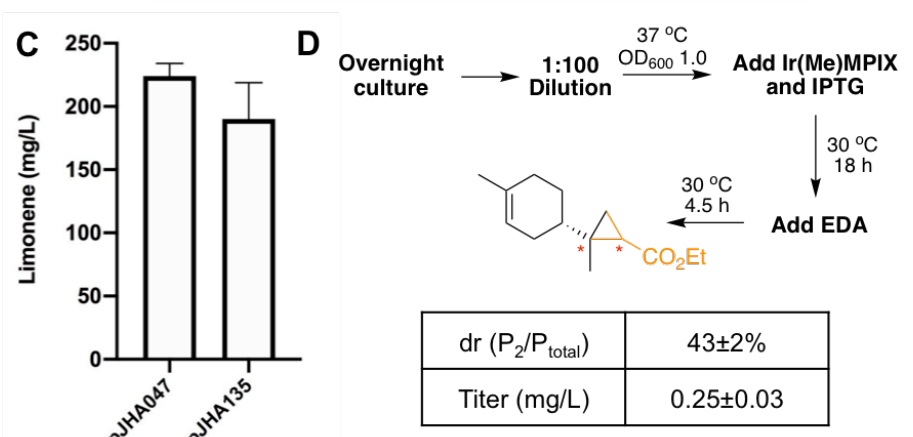

Fig. 3. (A) Ir-CYP119 in E. coli catalyzed the stereoselective cyclopropanation of exogenously added (-)-limonene in M9-rich growth media with diastereoselectivity distinct from that of the free cofactor and enhanced over that of reactions in vitro, indicating that the reaction in whole cells is catalysed by Ir-CYP119. (B) The heterologous pathway introduced into $E$. coli for the production of cyclopropyl limonene. The MTSA operon contains genes for the conversion of acetyl CoA to mevalonate; the MBI operon contains genes for the conversion of mevalonate to isoprenoid precursors; tr-GPPS: truncated sequence of geranyl pyrophosphate synthase; LS: limonene synthase.(28) (C) (-)-Limonene were produced with high titers in E. coli strains expressing the limonene pathway/hug operon (pJBEI6410+pJHA047) and limonene pathway/ hug operon/CYP119 (pJBEI6410+pJHA135) respectively. (D) The cyclopropyl limonene was produced stereoselectively from glucose in E. coli expressing limonene pathway/ hug operon/CYP119. P1-P4 are the four diastereomeric product numbered in the order of elution by GC. The selectivity of P1-P4 corresponds to its ratio in the total four diastereomeric products. $\mathrm{P}_{\text {total }}$ is the sum of four diastereomeric products. All data from whole-cell reactions are shown as the average from three biological repeats, with error bars indicating 1 standard deviation.

To combine terpene production and artificial catalysis, we transformed pJBEI6410 and pJHA135 into $E$. coli and induced protein expression simultaneously with addition of $\operatorname{Ir}(\mathrm{Me}) \mathrm{MPIX}$. The diazo compound was added after incubation of the culture overnight $(18 \mathrm{~h})$. Under these conditions, $250 \mu \mathrm{g} / \mathrm{L}$ of cyclopropyl limonene was produced after $4.5 \mathrm{~h}$ with diastereoselectivity $(43 \% \mathrm{dr}, 1.0: 3.2: 1.8: 1.5)$ that was within $10 \%$ of that of the reaction of exogenously added limonene in vivo (Fig. 3c). The similarity in the stereoselectivity shows that the cyclopropyl limonene is formed from the combination of the heterologous biosynthesis of limonene and cyclopropanation predominantly catalyzed by the ArM. 


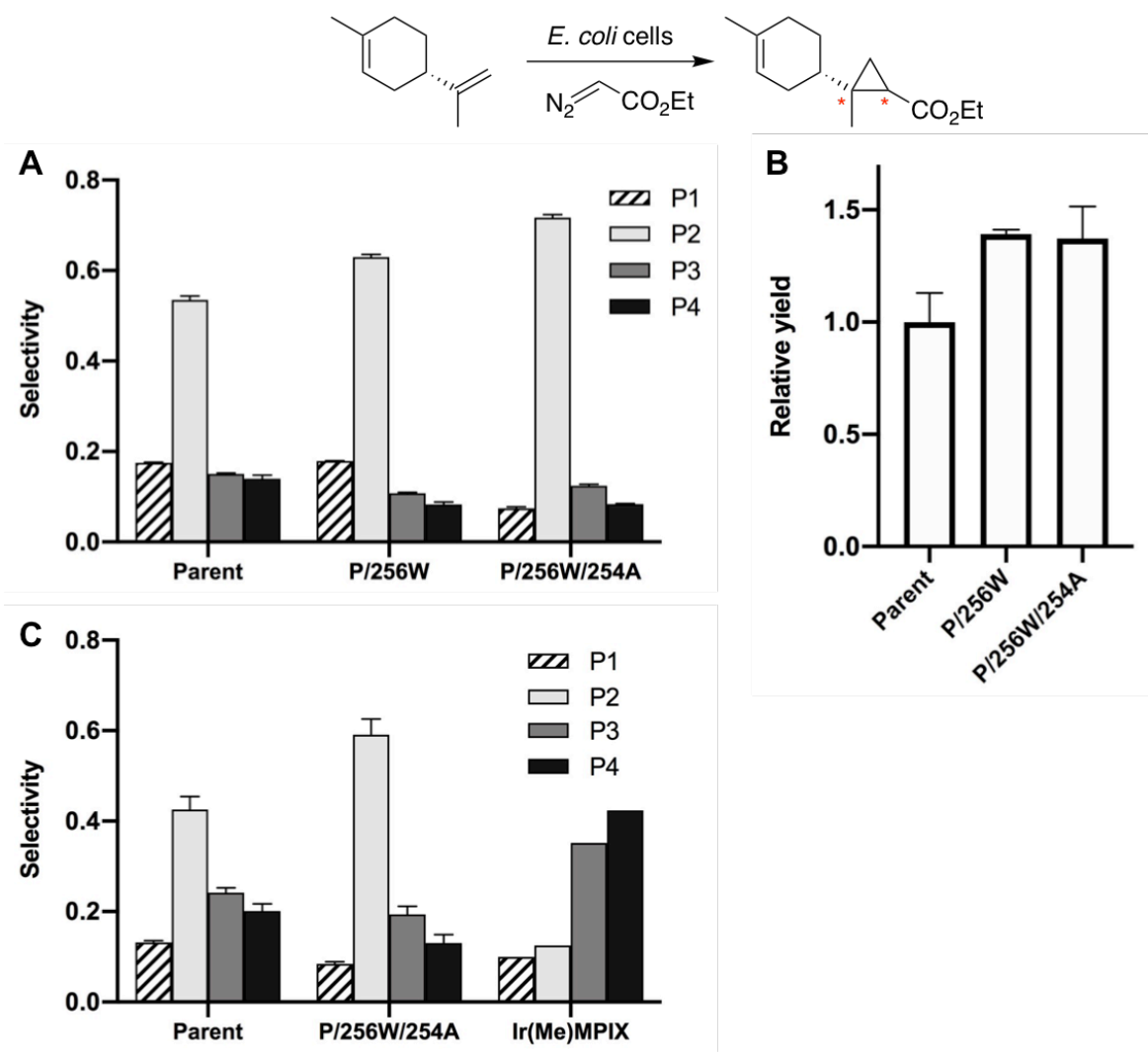

Fig. 4. Directed evolution of Ir-CYP119. (A) From whole-cell screening, mutations at R256 and V254 increased the stereoselectivity of the cyclopropanation of (-)-limonene. (B) The evolved mutants also catalyzed the cyclopropanations in higher yields. The relative yield is the amount of cyclopropyl limonene relative to that of the whole cell reaction with the parent mutant of CYP119. (C) By coupling the evolved mutant $(\mathrm{C} 317 \mathrm{G}, \mathrm{T} 213 \mathrm{G}, \mathrm{V} 254 \mathrm{~A}, \mathrm{~L} 155 \mathrm{~W}, \mathrm{R} 256 \mathrm{~W})$ with limonene biosynthesis, the diastereoselectivity of the cyclopropyl limonene from glucose in $E$. coli was higher than that with the pathway containing the parent mutant of CYP119 and distinct from that of the free cofactor. All data from biotransformations are shown as the average from three biological repeats, with error bars indicating 1 standard deviation.

To increase the stereoselectivity of the cyclopropanation of limonene, we constructed a library of CYP119 mutants from the triple-mutant scaffold containing additional changes to the amino acids around the binding site of the cofactor and tested their selectivity for cyclopropanation of limonene in whole cells (Fig. S7-S9). Previous directed evolution of Ir-CYP119 relied on purified protein variants or cell lysates. With a method to assemble functional Ir-CYP119 in vivo, the reactivity and selectivity of Ir-CYP119 mutants could be screened more rapidly. Initial screening at sites L69, F153, L205, A209, G210, T214, and R256 showed that the mutations at R256 increased the diastereoselectivity more than the mutations at the other positions. Saturation mutagenesis at this site showed that $\mathrm{P} / \mathrm{R} 256 \mathrm{~W}$ (P designates the triple mutant parent) catalyzed the cyclopropanation in higher yield and stereoselectivity $(63 \% \mathrm{dr}, 2.1: 7.6: 1.3: 1.0$; Fig. 4a, 4b) than did cells expressing the parent mutant. A second round of saturation mutagenesis at the neighboring V254 site showed that $\mathrm{P} / \mathrm{R} 256 \mathrm{~W} / \mathrm{V} 254 \mathrm{~A}$ reacted with an even higher stereoselectivity of $72 \% \mathrm{dr}(1.0$ : $9.6: 1.7: 1.1$; Fig. $4 \mathrm{a}$ and $4 \mathrm{~b}$ ). This mutant was introduced into the coupled strain to perform 
artificial biosynthesis with the more active and selective ArM. The titer and ratio of diasteromers of the product formed from this new pathway were $260 \mu \mathrm{g} / \mathrm{L}$ and $1.0: 7.0: 2.3: 1.5$, respectively (Fig. 4c).

This example of creating an artificial biosynthetic pathway in a microbial host that integrates both natural enzymes and an artificial P450 to produce new-to-nature products demonstrates the feasibility of creating organic molecules by combining synthetic biology with synthetic chemistry in vivo. One can envision this combination of biosynthesis and artificial metalloenzymes being applied to engineered pathways that produce unusual core structures, with enzymes that catalyze functionalizations of $\mathrm{C}-\mathrm{H}$ bonds to install abiotic groups where P450s might naturally operate, or with the artificial metalloenzyme operating in the middle of a pathway followed by diversification of the abiotic product with evolved downstream enzymes. One can even envision creating the reagents, like diazoesters, biosynthetically based on known pathways to these species. ${ }^{17}$ Such goals could be achieved in E. coli or in more complex organisms that produce diverse classes of natural products, such as polyketides or various alkaloids, thus opening a realm of biosynthesis beyond that limited by the scope of natural reactions.

Funding: This work was supported by the Department of Energy, Laboratory Directed Research and Development funding, under contract DE-AC01-05CH11231. ZL is an A*Star predoctoral fellow. We thank the College of Chemistry's NMR facility for resources provided and the staff for their assistance. Instruments in CoC-NMR are supported in part by the NIH (S10OD024998).

\section{References and Notes :}

1. Schwizer, F.; Okamoto, Y.; Heinisch, T.; Gu, Y.; Pellizzoni, M. M.; Lebrun, V.; Reuter, R.; Köhler, V.; Lewis, J. C.; Ward, T. R., Artificial Metalloenzymes: Reaction Scope and Optimization Strategies. Chemical Reviews 2018, 118 (1), 142-231.

2. (a) Gu, Y.; Natoli, S. N.; Liu, Z.; Clark, D. S.; Hartwig, J. F., Site-Selective Functionalization of $(\mathrm{sp} 3) \mathrm{C}-\mathrm{H}$ Bonds Catalyzed by Artificial Metalloenzymes Containing an Iridium-Porphyrin Cofactor. Angewandte Chemie International Edition 2019, 58 (39), 1395413960; (b) Hanna M. Key; Dydio, P.; Liu, Z.; Rha, J. Y.-E.; Nazarenko, A.; Seyedkazemi, V.; Clark, D. S.; Hartwig, J. F., Beyond Iron: Iridium-Containing P450 Enzymes for Selective Cyclopropanations of Structurally Diverse Alkenes. ACS Cent. Sci. 2017, 3 (4), 302-308; (c) Dydio, P.; Key, H. M.; Hayashi, H.; Clark, D. S.; Hartwig, J. F., Chemoselective, Enzymatic C-H Bond Amination Catalyzed by a Cytochrome P450 Containing an Ir(Me)-PIX Cofactor. Journal of the American Chemical Society 2017, 139 (5), 1750-1753.

3. (a) Chatterjee, A.; Mallin, H.; Klehr, J.; Vallapurackal, J.; Finke, A. D.; Vera, L.; Marsh, M.; Ward, T. R., An Enantioselective Artificial Suzukiase Based on the Biotin-Streptavidin Technology. Chem. Sci. 2016, 7, 673; (b) Abe, S.; Niemeyer, J.; Abe, M.; Takezawa, Y.; Ueno, T.; Hikage, T.; Erker, G.; Watanabe, Y., Control of the Coordination Structure of Organometallic Palladium Complexes in an apo-Ferritin Cage. Journal of the American Chemical Society 2008, 130 (32), 10512-10514.

4. $\quad$ (a) Letondor, C.; Pordea, A.; Humbert, N.; Ivanova, A.; Mazurek, S.; Novic, M.; Ward, T. R., Artificial Transfer Hydrogenases Based on the Biotin-(Strept)Avidin Technology: Fine Tuning the Selectivity by Saturation Mutagenesis of the Host Protein. J. Am. Chem. Soc. 2006, 128, 8320; (b) Skander, M.; Humbert, N.; Collot, J.; Gradinaru, J.; Klein, G.; Loosli, A.; Sauser, J.; Zocchi, A.; Gilardoni, F.; Ward, T. R., Artificial Metalloenzymes: (Strept)Avidin as Host for Enantioselective Hydrogenation by Achiral Biotinylated Rhodium-Diphosphine Complexes. $J$. 
Am. Chem. Soc. 2004, 126, 14411; (c) Lin, C. C.; Lin, C. W.; Chan, A. S. C., Catalytic Hydrogenation of Itaconic Acid in a Biotinylated Pyrphos-Rhodium(I) System in a Protein Cavity. Tetrahedron: Asymmetry 1999, 10, 1887.

5. (a) Philippart, F.; Arlt, M.; Gotzen, S.; Tenne, S. J.; Bocola, M.; Chen, H. H.; Zhu, L. L.; Schwaneberg, U.; Okuda, J., A Hybrid Ring-Opening Metathesis Polymerization Catalyst Based on an Engineered Variant of the Beta-Barrel Protein Fhua. Chem. - Eur. J. 2013, 19, 13865; (b) Lo, C.; Ringenberg, M. R.; Gnandt, D.; Wilson, Y.; Ward, T. R., Artificial Metalloenzymes for Olefin Metathesis Based on the Biotin-(Strept)Avidin Technology. Chem. Commun. 2011, 47, 12065; (c) Jeschek, M.; Reuter, R.; Heinisch, T.; Trindler, C.; Klehr, J.; Panke, S.; Ward, T. R., Directed evolution of artificial metalloenzymes for in vivo metathesis. Nature 2016, 537 (7622), 661-665.

6. Zhao, J.; Rebelein, J. G.; Mallin, H.; Trindler, C.; Pellizzoni, M. M.; Ward, T. R., Genetic Engineering of an Artificial Metalloenzyme for Transfer Hydrogenation of a Self-Immolative Substrate in Escherichia coli's Periplasm. Journal of the American Chemical Society 2018, 140 (41), 13171-13175.

7. (a) Grimm, A. R.; Sauer, D. F.; Polen, T.; Zhu, L.; Hayashi, T.; Okuda, J.; Schwaneberg, U., A Whole Cell E. coli Display Platform for Artificial Metalloenzymes: Poly(phenylacetylene) Production with a Rhodium-Nitrobindin Metalloprotein. ACS Catalysis 2018, 8 (3), 2611-2614; (b) Heinisch, T.; Schwizer, F.; Garabedian, B.; Csibra, E.; Jeschek, M.; Vallapurackal, J.; Pinheiro, V. B.; Marlière, P.; Panke, S.; Ward, T. R., E. coli surface display of streptavidin for directed evolution of an allylic deallylase. Chemical Science 2018, 9 (24), 5383-5388.

8. (a) Khanna, N.; Esmieu, C.; Mészáros, L. S.; Lindblad, P.; Berggren, G., In vivo activation of an [FeFe] hydrogenase using synthetic cofactors. Energy \& Environmental Science 2017, 10 (7), 1563-1567; (b) Song, W. J.; Tezcan, F. A., A designed supramolecular protein assembly with in vivo enzymatic activity. Science 2014, 346 (6216), 1525.

9. Huang, W.; Wilks, A., Extracellular Heme Uptake and the Challenge of Bacterial Cell Membranes. Annual Review of Biochemistry 2017, 86 (1), 799-823.

10. Brahmkshatriya, P. P.; Brahmkshatriya, P. S., Terpenes: Chemistry, Biological Role, and Therapeutic Applications. In Natural Products: Phytochemistry, Botany and Metabolism of Alkaloids, Phenolics and Terpenes, Ramawat, K. G.; Mérillon, J.-M., Eds. Springer Berlin Heidelberg: Berlin, Heidelberg, 2013; pp 2665-2691.

11. Helfrich, E. J. N.; Lin, G.-M.; Voigt, C. A.; Clardy, J., Bacterial terpene biosynthesis: challenges and opportunities for pathway engineering. Beilstein Journal of Organic Chemistry 2019, 15, 2889-2906.

12. (a) Dydio, P.; Key, H. M.; Nazarenko, A.; Rha, J. Y. E.; Seyedkazemi, V.; Clark, D. S.; Hartwig, J. F., An Artificial Metalloenzyme with the Kinetics of Native Enzymes. Science 2016, 354, 102; (b) Key, H. M.; Dydio, P.; Clark, D. S.; Hartwig, J. F., Abiological Catalysis by Artificial Haem Proteins Containing Noble Metals in Place of Iron. Nature 2016, 534, 534.

13. (a) Lelyveld, V. S.; Brustad, E.; Arnold, F. H.; Jasanoff, A., Metal-Substituted Protein MRI Contrast Agents Engineered for Enhanced Relaxivity and Ligand Sensitivity. Journal of the American Chemical Society 2011, 133 (4), 649-651; (b) Bordeaux, M.; Singh, R.; Fasan, R., Intramolecular $\mathrm{C}(\mathrm{sp} 3) \mathrm{H}$ amination of arylsulfonyl azides with engineered and artificial myoglobin-based catalysts. Bioorganic \& Medicinal Chemistry 2014, 22 (20), 5697-5704; (c) Reynolds, E. W.; Schwochert, T. D.; McHenry, M. W.; Watters, J. W.; Brustad, E. M., Orthogonal Expression of an Artificial Metalloenzyme for Abiotic Catalysis. ChemBioChem 2017, 18 (24), 2380-2384. 
14. Henderson, D. P.; Wyckoff, E. E.; Rashidi, C. E.; Verlei, H.; Oldham, A. L., Characterization of the $<\mathrm{em}>$ Plesiomonas shigelloides $</ \mathrm{em}>$ Genes Encoding the Heme Iron Utilization System. Journal of Bacteriology 2001, 183 (9), 2715-2723.

15. Ascue Avalos, G. A.; Toogood, H. S.; Tait, S.; Messiha, H. L.; Scrutton, N. S., From Bugs to Bioplastics: Total (+)-Dihydrocarvide Biosynthesis by Engineered Escherichia coli. ChemBioChem 2019, 20 (6), 785-792.

16. Alonso-Gutierrez, J.; Chan, R.; Batth, T. S.; Adams, P. D.; Keasling, J. D.; Petzold, C. J.; Lee, T. S., Metabolic engineering of Escherichia coli for limonene and perillyl alcohol production. Metabolic Engineering 2013, 19, 33-41.

17. Waldman, A. J.; Balskus, E. P., Discovery of a Diazo-Forming Enzyme in Cremeomycin Biosynthesis. The Journal of Organic Chemistry 2018, 83 (14), 7539-7546. 